\title{
Pandemic (H1N1) 2009: a clinical spectrum in the general paediatric population
}

\author{
Penelope Jane Larcombe, ${ }^{1}$ Susan Evelyn Moloney, ${ }^{1}$ Peter Alan Schmidt ${ }^{1}$
}

${ }^{1}$ Gold Coast Hospital, Gold Coast Australia, Queensland, Australia

Correspondence to

Dr Penelope Jane Larcombe, Gold Coast Hospital,

108 Nerang Street,

Southport,

Queensland 4215,

Australia;

penny_larcombe@health.qld gov.au

Accepted 29 0ctober 2009 Published Online First 10 November 2009

\begin{abstract}
This retrospective case series describes the clinical spectrum of 43 children with pandemic (H1N1) 2009 admitted to a single hospital in Australia during the peak winter flu season. Clinical features, diagnoses, length of hospitalisation and complications were reviewed in children up to 17 years of age with proven pandemic (H1N1) 2009 by RT-PCR. The median age was 6 years, $42 \%$ had a pre-existing medical condition. The most common presentation was fever and cough, and $88 \%$ of patients met our criteria for flu-like illness. Consolidation on chest $x$ ray was the most common diagnosis $(n=20$, $46 \%)$, followed by dehydration $(n=13,30 \%)$. Three (7\%) had encephalopathy and two (5\%) had diabetic ketoacidosis. There were two intensive care admissions and no deaths. Pandemic (H1N1) 2009 flu has a wide range of presentation in the paediatric population. The diagnosis should be considered during the current pandemic in any child with fever, or who is unwell.
\end{abstract}

\section{INTRODUCTION}

Since being identified in Mexico in April 2009, human influenza A (H1N1) virus has spread across the world and has widespread community transmission in many countries. The World Health Organization escalated the pandemic to alert phase 6 in early June 2009. ${ }^{1}$ The outbreak coincided with Australia's flu season resulting in 35579 confirmed cases of pandemic (H1N1) 2009 and 161 deaths, in Australia as of 7 September $2009 .^{2}$ An early report of 18 hospitalised patients of the initial outbreak in Mexico showed that the pandemic (H1N1) 2009 virus caused severe illness and death in previously healthy young to middleaged persons. ${ }^{3}$ Despite this, it has been found that the majority of patients continue to experience mild illness.

The purpose of this study was to describe the clinical spectrum of pandemic (H1N1) 2009 virus in the general paediatric population. The Gold Coast Hospital serves a population of over 455000 people. Our case series describes the epidemiological characteristics, clinical features, range of diagnoses and length of hospitalisation in 43 children positive for pandemic (H1N1) 2009 admitted to the Gold Coast Hospital.

\section{METHODS}

This retrospective study was conducted by review of medical charts, laboratory and radiological findings of all children admitted to the Gold Coast Hospital with confirmed pandemic (H1N1) 2009. The study period was from the 25 May 2009 to the 16 August 2009, coinciding with Australia's flu season. During this period, all children admitted into hospital with a febrile or respiratory illness were tested for pandemic (H1N1) 2009 by two Taqman based real-time RT-PCR methods, designed locally in Australia. The two assays, H1-PCR and N1-PCR, were designed targeting the pandemic (H1N1) 2009 virus haemagglutinin and neuraminidase genes, respectively. These RT-PCR methods are found to be sensitive and specific for pandemic (H1N1) 2009 RNA. ${ }^{4}$ The tests were done at the closest tertiary centre $1 \mathrm{~h}$ away and took $2 \mathrm{~h}$ to complete. In the peak of the pandemic, due to the overwhelming number of samples, it took $48 \mathrm{~h}$ for results to return. Specimens were collected from nasal pharyngeal aspirates or nasal pharyngeal swabs. These specimens were also tested with a multiplex PCR assay for a select respiratory viral panel including influenza $A$, influenza $B$, respiratory syncytial virus, parainfluenza types 1,2 and 3 , adenovirus and human metapneumovirus.

Epidemiological characteristics observed were age, gender and pre-existing medical conditions. Pre-existing medical conditions were obtained from the initial history and chart review. These were then categorised according to physiological system. Asthmatic patients were included if they were, at the time of admission, on preventive treatment for asthma. Due to variable documentation of obesity or indigenous background in the medical charts, these factors were not examined in our study.

Clinical features were obtained from findings documented by the treating physician. Based on Queensland Health's pandemic (H1N1) 2009 case definition, children had flu-like illness if they presented with a fever of at least $38^{\circ} \mathrm{C}$, or a history of fever, with one symptom of cough, sore throat or rhinorrhoea. ${ }^{5}$

Diagnosis was based on history and examination findings documented by the admitting physician, laboratory and radiological findings. In our study, respiratory diagnoses were divided into three groups namely, asthma, bronchiolitis and consolidation on chest $x$ ray. Asthma was defined as the presence of wheezing and/or chest retraction in those with a past history of asthma, without evidence of consolidation on chest $x$ ray. Bronchiolitis was defined as the presence of wheezing and/or chest retraction in those $<1$ year of age without evidence of consolidation on chest $x$ ray. Due to the difficulty in differentiating consolidation caused by pneumonia or asthma, those with bilateral or unilateral consolidation on chest $x$ ray were analysed in one group called consolidation. Febrile convulsion was defined as a seizure associated with a fever, or history of recent fever, with no previous history of afebrile seizures or 
evidence of central nervous infection or metabolic abnormality. Dehydration was defined according to the admitting physician's initial assessment based on history of fluid loss, or poor oral intake, clinical signs including tachycardia, dry mucous membranes, poor central capillary refill, poor urine output and laboratory findings. Encephalopathy was defined as altered mental status lasting greater than $24 \mathrm{~h}$. Diabetic ketoacidosis was defined by biochemical criteria of a blood glucose greater than $11 \mathrm{mmol} /$ litre and evidence of metabolic acidosis (venous $\mathrm{pH}<7.3$ and/or plasma bicarbonate $<15 \mathrm{mmol} /$ litre).

Patients were treated with the antiviral, oseltamivir, at the discretion of the admitting physician. Factors considered when commencing treatment were based on the joint position statement published by the Australasian Society for Infectious Diseases (ASID) and the Thoracic Society of Australia and New Zealand (TSANZ). ${ }^{6}$ These recommendations included treatment for children with risk factors for severe disease, particularly children $<5$ years of age, those with immunosuppression or chronic disease like asthma, cardiorespiratory disease, diabetes and renal failure. In children with no risk factors for severe disease, oseltamivir was recommended in those who presented within $48 \mathrm{~h}$ of onset of symptoms. Oseltamivir was not recommended in those $<1$ year of age due to limited safety data. Oseltamivir was given to those hospitalised with severe flu infection even after $48 \mathrm{~h}$ of symptom onset.

\section{RESULTS}

In the period from the end of May to mid-August 2009, a total of 683 children were admitted to hospital. Of these 130 children were screened, via nasopharyngeal swab or aspirate, for pandemic (H1N1) 2009 influenza virus and other common respiratory viruses on our respiratory viral PCR panel. Fortythree children were proven to have pandemic (H1N1) 2009. The characteristics of the patients with pandemic (H1N1) 2009 are listed in table 1.

Fever was the most common presenting symptom ( $n=41$, $95 \%)$ followed by cough ( $\mathrm{n}=40,93 \%)$. Other common symptoms were vomiting $(n=21,49 \%)$, rhinorrhoea $(n=19,44 \%)$ and respiratory distress $(\mathrm{n}=16,37 \%)$. Headache, myalgia, sore throat, lethargy, abdominal pain, diarrhoea, confusion and seizures were other symptoms reported at presentation. Two $(5 \%)$ patients did not have a fever or history of fever. Both had pre-existing medical conditions. Thirty-eight (88\%) patients fulfilled our criteria for flu-like illness.

The most common diagnosis was consolidation on chest $\mathrm{x}$-ray, with a total of $20(46 \%)$ patients. Thirteen $(30 \%)$ patients were clinically dehydrated, and four (9\%) patients had an exacerbation of asthma. Encephalopathy was diagnosed in three $(7 \%)$ patients and two $(5 \%)$ were admitted with diabetic ketoacidosis. Febrile convulsion, bronchiolitis and abdominal pain each occurred in one (2\%) patient.

Overall, the median length of stay was 2 days. Those with consolidation had a median length of stay of 2 days while asthma, bronchiolitis, clinical dehydration and febrile convulsion had a median length of 1 day. Patients with encephalopathy and diabetic ketoacidosis had a median length of stay of 4 and 5 days, respectively. Those who were previously healthy had a median stay of 1 day while those who had a pre-existing medical condition had a median stay of 2 days. Twenty-nine $(67.4 \%)$ patients were treated with oseltamivir.

Of the 43 patients, there were no deaths. Two patients (4.6\%) required intensive care; both had pre-existing disorders. One patient had severe bilateral pneumonia and respiratory distress
Table 1 Patient demographics $(\mathrm{N}=43)$

\begin{tabular}{lc}
\hline Variable & Value \\
\hline Male sex, n (\%) & $22(51.2)$ \\
Age, median & $6(0.25-17)$ \\
$\quad$ Overall, years (range) & 5 \\
$\quad$ Patients previously healthy, years & 11 \\
$\quad$ Patients with pre-existing condition, years & \\
Age group, n (\%) & $4(9.3)$ \\
$\quad<1$ years & $14(32.6)$ \\
$\quad$ 6-5 years & $13(30.2)$ \\
$\quad 13-17$ years & $12(27.9)$ \\
Patients with pre-existing condition, overall total $n(\%)$ & $18(41.9)$ \\
Respiratory, total & $10(23.3)$ \\
Asthma on preventer & $8(18.6)$ \\
Cystic fibrosis & $1(2.3)$ \\
Chronic neonatal lung disease & $1(2.3)$ \\
Congenital cardiac disease & $2(4.6)$ \\
Immunosuppression, total & $3(7.0)$ \\
Oncology & $2(4.6)$ \\
Autoimmune hepatitis & $1(2.3)$ \\
Metabolic/endocrine, total & $3(7.0)$ \\
Diabetes & $1(2.3)$ \\
Congenital adrenal hypoplasia & $1(2.3)$ \\
Neuromuscular, total & $1(2.3)$ \\
Rerebral palsy & $2(4.6)$ \\
\hline & $1(2.3)$ \\
$\quad$ & $1(2.3)$ \\
\hline
\end{tabular}

requiring non-invasive ventilation with a background of Retts syndrome. The other patient was admitted to intensive care in severe diabetic ketoacidosis. Five patients (11.6\%) were readmitted with complications, including secondary pneumonia, ongoing confusion and exacerbation of asthma. Three of these patients had been treated initially with oseltamivir.

\section{DISCUSSION}

Our case series of 43 paediatric patients with proven pandemic (H1N1) 2009 admitted to hospital during the peak of Australia's flu season demonstrates the wide range of presentations in the paediatric population. All paediatric age groups were affected and almost half of those admitted had an underlying medical problem.

During this study period, there was a low clinical threshold to obtain pandemic (H1N1) 2009 identification. It became apparent that children were being admitted with a wide range of presentations. Fever and cough were present in almost all our cases. Screening only with our criteria for flu-like illness would have missed $12 \%$ of children with pandemic (H1N1) 2009 in contrast to a similar report in the UK which found it would have missed $40 \%$ of cases using their case definition which required fever and two other symptoms. ${ }^{7}$ This same report found that $19 \%$ of cases did not have a temperature of at least $38^{\circ} \mathrm{C}$ or history of fever in contrast to our study which found only $5 \%$. A full screening study of all admissions may be warranted in the future. This was unable to be done due to the significant impact it would have on the testing laboratory during a pandemic.

Consolidation on chest $x$ ray was the most common diagnosis followed by clinical dehydration requiring rehydration in hospital. Interestingly three patients had encephalopathy. Similar neurological complications have been documented in patients with pandemic (H1N1) 2009 in Dallas, Texas as well 
as in other seasonal flu epidemics. ${ }^{8}$ In one case, a 16-year-old boy with no previous medical or psychological disorder, had hallucinations and paranoid delusions for 7 days. The other two cases, 2 and 9 years of age, had seizures and altered mental status. Two patients were admitted with diabetic ketoacidosis, one with known diabetes and the other their first presentation.

\section{CONCLUSION}

Pandemic (H1N1) 2009 in the general paediatric population can have a wide range of presentation. Respiratory illness was the most common presentation, there were however, less typical presentations like encephalopathy and diabetic ketoacidosis. There were no deaths and two admissions to intensive care in which both patients had pre-existing medical conditions. Pandemic (H1N1) 2009 should be considered as a diagnosis in any child with fever or who is unwell, even without fever.

Acknowledgements We would like to acknowledge Ms Ping Zhang for assistance with statistics.
Competing interests None.

Provenance and peer review Not commissioned; externally peer reviewed.

\section{REFERENCES}

1. World Health Organization. New influenza A (H1N1) virus: global epidemiological situation June 2009. Wkly Epidemiol Rec 2009;84:249-60.

2. Australian Government Department of Health and Ageing. Pandemic (H1N1) 2009 update bulletin. www.healthemergency.gov.au/ (Accessed September 2009).

3. Perez-Padilla R, de la Rosa-Zamboni D, Ponce de Leon S, et al. Pneumonia and respiratory failure from swine-origin influenza $\mathrm{A}$ (H1N1) in Mexico. N Eng/ J Med 2009;361:680-9.

4. Whiley DM, Bialasiewicz S, Bletchly C, et al. Detection of novel influenza A(H1N1) virus by real-time RT-PCR. J Clin Virol 2009;45:203-4.

5. Queensland Health. Queensland swine flu response. www.health.qld.gov.au/ swineflu/html/faqs.asp\# symptoms (Accessed August 2009).

6. Cheng AC, Dwyer DE, Kotsimbos AT, et al. ASID/TSANZ guidelines: treatment and prevention of H1N1 influenza 09 (human swine influenza) with antiviral agents. MJA 2009;19:1-8.

7. Hackett $\mathbf{S}$, Hill L, Patel J, et al. Clinical characteristics of paediatric H1N1 admissions in Birmingham, UK. Lancet 2009:374:605.

8. Evan AS, Agadi S, Siegal JD, et al. Neurologic complications associated with novel influenza A (H1N1) virus infection in children. MMWR 2009;58:773-8. 